\title{
Early and Long-Term Outcomes after Direct Bridge-to-Transplantation with Extracorporeal Membrane Oxygenation
}

\author{
Mélanie Hébert, MD, MSc, ${ }^{1}$ Pierre-Emmanuel Noly, MD, PhD ${ }^{1}$ Yoan Lamarche, MD, MSc, ${ }^{1}$ \\ Ismail Bouhout, $\mathrm{MD}, \mathrm{MSc},{ }^{1}$ Marion Mauduit, $\mathrm{MD},{ }^{2}$ Geneviève Giraldeau, $\mathrm{MD},{ }^{3}$ Bernard Lelong, $\mathrm{MD},{ }^{2}$ \\ Jean-Philippe Verhoye, $\mathrm{MD}, \mathrm{PhD},{ }^{2}$ Michel Carrier, $\mathrm{MD}, \mathrm{MBA},{ }^{1}$ Erwan Flecher, $\mathrm{MD}, \mathrm{PhD}^{2}$ \\ ${ }^{1}$ Department of Cardiac Surgery, Montreal Heart Institute - Université de Montréal, 5000 Bélanger Street, Montréal, Canada; \\ ${ }^{2}$ Department of Thoracic and Cardiovascular Surgery, Rennes Hospital, University of Rennes 1, 2 rue Henri Le Guilloux, 35000 \\ Rennes, France; ${ }^{3}$ Department of Cardiology, Montreal Heart Institute - Université de Montréal, 5000 Bélanger Street, Montréal, \\ Canada
}

\section{ABSTRACT}

Background: Veno-arterial extracorporeal membrane oxygenation (VA-ECMO) as direct bridge-to-transplantation (dBTT) remains controversial. We compared the short- and long-term outcomes of adult patients undergoing urgent heart transplantation (HT) with (dBTT) and without (nonBTT) VA-ECMO support at the time of HT.

Methods: Adults who underwent urgent HT in two institutions were assessed $(N=133$; dBTT: $N=34$ and non-BTT: $N=99$ ). Patient characteristics, donor characteristics, inhospital outcomes, and overall survival were compared. Mean follow up was $77 \pm 38$ months and was $100 \%$ complete. Mortality predictors were identified using univariate and multivariate analyses.

Results: Before HT, patients with dBTT had higher rates of ischemic cardiomyopathy, acute kidney injury, liver failure, respiratory failure, and longer graft ischemia times. More patients in the dBTT group had complications, such as requiring VA-ECMO postoperatively ( $\mathrm{dBTT}=50 \%$ vs. nonBTT $=20 \%, P<0.01)$. Hospital deaths (dBTT $=23 \%$ vs. nonBTT $=19 \%, P=0.58)$, one-year $(74 \%$ vs. $80 \%)$ and five-year survival ( $62 \%$ vs. $75 \%, P=0.74$ for overall survival) were not significantly different. The MELD-XI score and previous cardiac surgery were independent predictors of hospital mortality.

Conclusion: Direct bridge-to-transplantation in patients on VA-ECMO support was not associated with worse longterm outcomes compared with non-VA-ECMO urgent HT, especially in recipients without any associated organ failure and a low MELD-XI score before HT.

Received April 1, 2021; received in revised form April 12, 2021; accepted April 13, 2021.

Correspondence: Pierre-Emmanuel Noly, MD, PbD, Department of Cardiac Surgery, Montreal Heart Institute, 5000 Bélanger Street, Montréal, Canada, H1T 1C8, Telephone 514 373-3330, Fax $514593-2157$ (e-mail: noly. pierreemmanuel@gmail.com).

\section{INTRODUCTION}

Direct bridge-to-transplantation (dBTT) might be a longterm option for patients supported with veno-arterial extracorporeal membrane oxygenation (VA-ECMO) in the absence of myocardial recovery. This strategy is currently used in $1 \%$ to $10 \%$ of heart transplantation (HT), varying by country and by heart graft allocation policy [Agence de la Biomédecine 2016; Jasseron 2016; The International Society for Heart and Lung Transplantation 2018]. The use of VA-ECMO for cardiogenic shock has dramatically increased over the last decade [McCarthy 2015; Mandawat 2017; Ouyang 2018; Sauer 2015] and about $4 \%$ to $24 \%$ of these patients would be a candidate for dBTT [Paden 2013]. In most countries, HT candidates supported by VA-ECMO receive the highest priority. As the number of patients on VA-ECMO increases, the number of HT after dBTT is expected to increase, too. However, dBTT remains a debated strategy in the context of the worldwide organ shortage and raises many concerns about post-HT outcomes and survival [Dardas 2018]. On one hand, several publications reported that post-HT survival in these critically ill patients is lower than in other HT candidates, but on other hand, HT provides a potential survival benefit in patients supported by VA-ECMO. Some authors also reported acceptable short- and mid-term outcomes in well-selected patients using this strategy [Barth 2012; Cho 2015]. There is therefore a need for short- and long-term survival data after dBTT to improve patient selection and to better understand the clinical impact of dBTT.

In this study, we aimed to compare the recipient characteristics, early outcomes, and long-term survival of adult patients who underwent HT after dBTT with VA-ECMO to those undergoing urgent HT without previous VA-ECMO in two institutions.

\section{METHODS}

Study population: This study adheres to the tenets of the 1975 Declaration of Helsinki and was approved by the Institutional Review Board of the two participating institutions, who 
waived the need for individual patient consent. We reviewed all adults $(N=296)$ who underwent orthotopic HT at the Rennes University Hospital (Rennes, France) $(N=161)$ and at the Montreal Heart Institute (MHI, Montreal, Canada) ( $N$ $=135$ ) between January 2003 and December 2013. Exclusion criteria were non-urgent HT $(N=147)$, re-transplantation $(N=5)$, urgent HT for left ventricular assist device (LVAD) complications $(N=8)$, and combined heart-kidney transplantation $(N=3)$. The remaining patients who underwent urgent HT $(N=133)$ were included and divided into two groups: the dBTT group $(N=34)$ included patients supported by VAECMO at the time of HT and the non-BTT group $(N=99)$ included patients who had urgent HT without previous VAECMO. (Supplementary Figure 1) Urgent HT was defined in our study as a transplantation with priority, for candidates with a high risk of death without transplantation. It corresponds to statuses 3.5 , or 4 and some status 3 at the MHI and to type-1 super-emergency at the Rennes Hospital. Demographic, clinical, biological, and echocardiographic characteristics before and after HT were compared between the dBTT and non-BTT groups. All data retrospectively were obtained from patient charts, local digital database systems, and the database of the Agence de la biomédecine ("Cristal" database). The mean follow-up was $77 \pm 38$ months and was $100 \%$ complete.

Cardiac graft allocation in France and Canada: Allocation systems in France and Canada nationally prioritize the candidates with the highest risk of early death without transplantation. In France, the highest priority status (type 1 super-emergency) was granted to the sickest patients, defined as patients on inotropes with in-hospital monitoring (i.e.

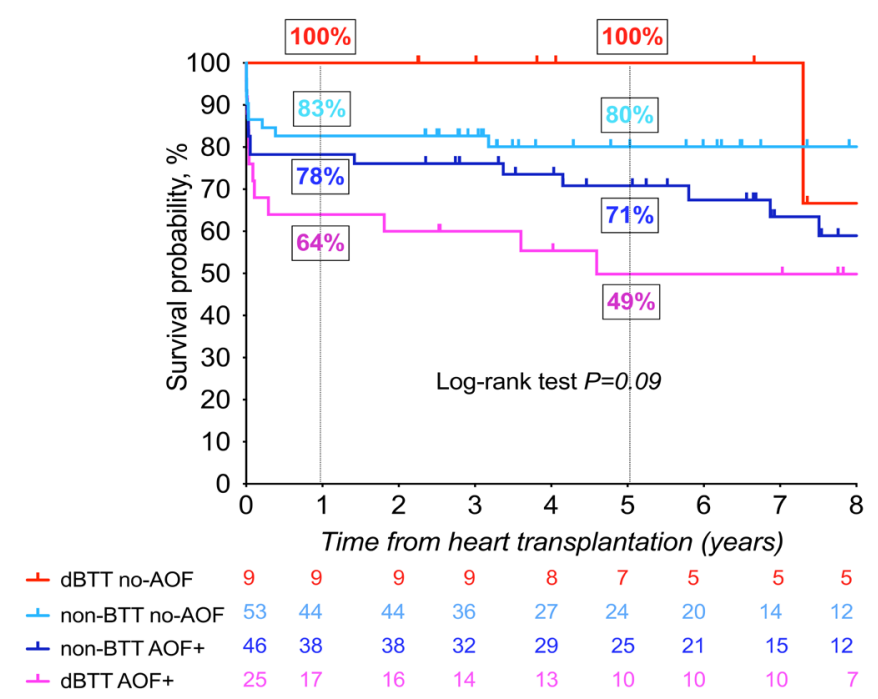

Figure 1. Kaplan-Meier curves presenting postoperative survival in the direct bridge-to-transplantation (dBTT) and the non-BTT groups, according to the presence $\left(\mathrm{AOF}^{+}\right.$) or absence (non-AOF) of associated organ failure (AOF) before heart transplantation. AOF was defined as at least one of the following: acute kidney injury (>50\% increase in glomerular filtration rate), liver failure (MELD-XI score > 16.4), or respiratory failure (need for mechanical ventilation). excluding home infusion inotrope therapy patients) and/or supported by a short-term mechanical circulatory support (MCS), such as VA-ECMO. Priority is not granted to stable LVAD patients without device-related complications. In the absence of a donor, this status expires after a maximum of four days, and patients then have regional prioritization. In Canada, patients with VA-ECMO also are listed with the highest priority status (status 4) without a time limit, though they must be recertified every seven days as a status 4 by a qualified physician, if it is still medically appropriate. Priority also is given to hospitalized patients on inotropes who are listed as statuses 3 or 3.5 [Haddad 2009; Isaac 2011].

Definitions and outcomes: Refractory cardiogenic shock was defined as a state of critical end-organ hypoperfusion, due to reduced cardiac output, despite optimal medical treatment [Levy 2015]. Associated organ failures (AOF) before HT were defined as follows: acute kidney injury as an acute decrease in glomerular filtration rate of $>50 \%$ [Mehta 2007]; respiratory failure as the need for mechanical ventilation; acute liver failure as a Model for End-stage Liver Disease excluding INR (MELD-XI) score $>16.4$ (MELD XI score $=$ $5.11 \times \ln ($ serum bilirubin $)+11.76 \times \ln$ (serum creatinine $)+$ 9.44) [Grimm 2015].

The primary outcome was five-year survival after HT. Secondary outcomes were all-cause in-hospital mortality, one-year and ten-year survival, and postoperative complications (i.e. primary graft dysfunction, right ventricular failure, need for mechanical circulatory support or temporary dialysis, acute kidney injury, disabling stroke, acute rejection, and sternal complications).

Indications and use of VA-ECMO: In both institutions, VA-ECMO is the device of choice to assist patients with severe refractory cardiogenic shock and is used as a bridge-to-recovery, bridge-to-candidacy, or bridge-to-transplantation. There are about 70 VA-ECMO per year at Rennes Hospital and 25 at the MHI. In both institutions, VA-ECMO is considered in patients showing evidence of end-organ hypoperfusion, low cardiac index $\left(<2.2 \mathrm{l} / \mathrm{min} / \mathrm{m}^{2}\right)$ despite adequate intravascular volume, low left ventricular ejection fraction and/or need for increasing doses of inotropes (e.g. epinephrine $>1 \mu \mathrm{g} / \mathrm{kg} / \mathrm{min}$, dobutamine $>20 \mu \mathrm{g} / \mathrm{kg} / \mathrm{min}$ and $/$ or norepinephrine $>1 \mu \mathrm{g} / \mathrm{kg} /$ min) with increasing acidosis and lactate levels. Patients who

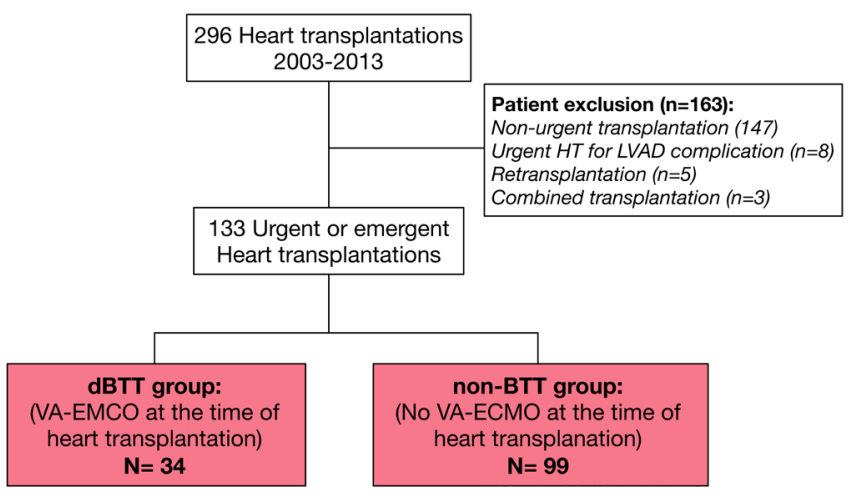

Supplementary Figure 1. Flowchart of the study population. 
Table 1. Comparison of recipient characteristics before heart transplantation

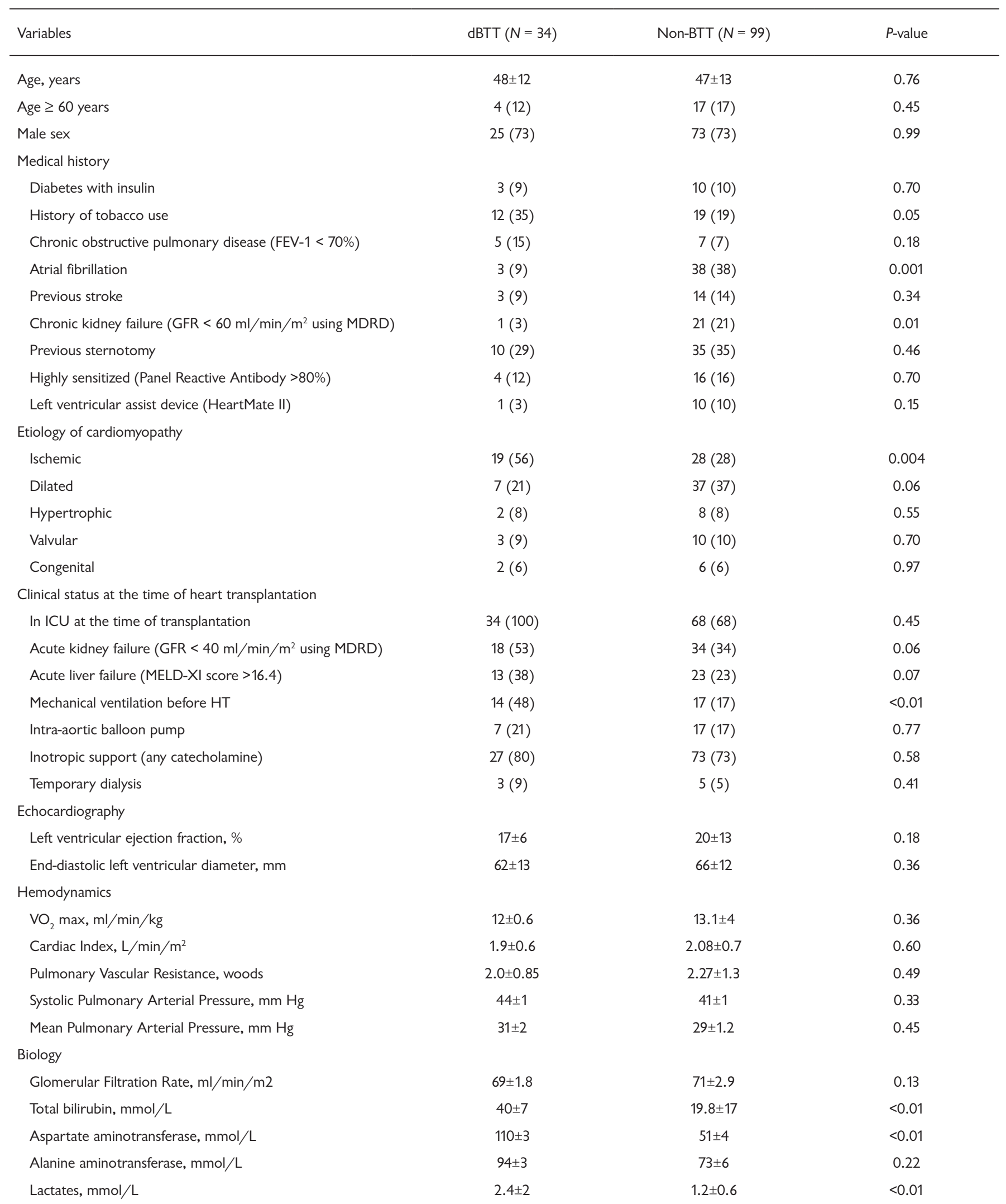




\begin{tabular}{lccc} 
NT-proBNP, ng/L & $5015 \pm 3990$ & $5014 \pm 5671$ & 0.75 \\
MELD XI score & $14.4 \pm 5$ & $13.7 \pm 4$ & 0.05 \\
Operative data & & $132 \pm 64$ & $<0.01$ \\
Cardiopulmonary bypass time, minutes & $164 \pm 50$ & $126 \pm 53$ & 0.12 \\
Cross-clamp time, minutes & $113 \pm 35$ & \\
\hline
\end{tabular}

Data are mean \pm SD or $\mathrm{n}(\%)$. FEV-1, forced expiratory volume in 1 second; GFR, glomerular filtration rate; MDRD, Modification of Diet in Renal Disease; MELD XI score: $5.11 \times \ln ($ serum bilirubin) $+11.76 \times \ln ($ serum creatinine $)+9.44$

Supplementary Table 1. Comparison of donor characteristics

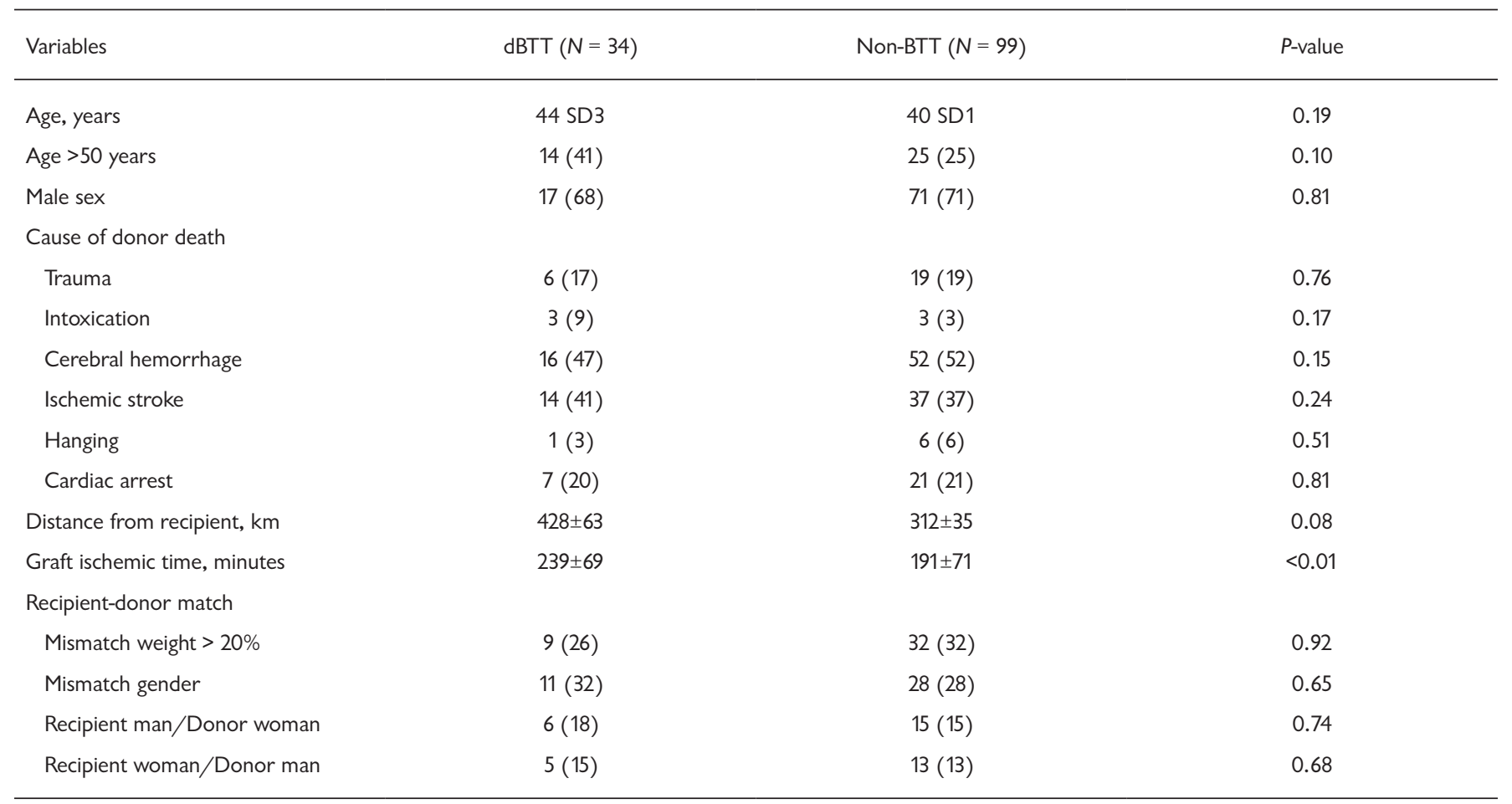

Data are mean \pm SD or $\mathrm{n}(\%)$

continue to deteriorate despite being dependent of inotropic support or are intolerant of inotropes also will be considered for VA-ECMO support. Patients with poor life expectancy and a severe, irreversible underlying condition are not deemed candidates for VA-EMCO [Muller 2016].

In this series, five patients (15\%) were Interagency Registry of Mechanically Assisted Circulatory Support (INTERMACS) 1 , and 29 patients (85\%) were INTERMACS 2 before VA-ECMO implantation. After implantation, ventricular function recovery was assessed daily using arterial pressure waveform, left ventricular outflow tract velocity time integral, and echocardiographic left ventricular ejection fraction. In the absence of recovery after 48 to 72 hours, a pre-transplantation workup is initiated. The duration of VA-ECMO support before waiting list registration varied according to the underlying cause of the cardiogenic shock and to the recovery potential. Nine patients $(26 \%)$ already were listed at the time of VA-ECMO implantation, and 25 patients (74\%) were listed after $4.5 \pm 5$ days on circulatory support. The mean time between VA-ECMO implantation and HT was $6.7 \pm 1$ days. In this series, the underlying etiologies were acute decompensation on chronic heart failure $(N=16,47 \%)$, acute myocardial infarction $(N=6,18 \%)$, refractory ventricular arrhythmia $(N$ $=6,18 \%)$, cardiac arrest $(N=5,15 \%)$, and myocarditis $(N$ $=1,3 \%)$. Sedation was stopped before HT, and all patients had to have an adequate neurological evaluation (i.e. normal clinical exam and head computed tomography). Whenever possible, patients under VA-ECMO support were extubated, and the aim was to achieve satisfactory associated organ injury recovery prior to HT. Among the dBTT group, 25 patients (73\%) had at least one AOF before HT compared with 48 patients $(48 \%)$ in the non-BTT group $(P=0.01)$. Registration on the waiting list with a priority status was discussed by a local heart team and had to be validated by a national expert or committee.

A surgical peripheral access (i.e. femoro-femoral) was 
used for all patients in the dBTT group. An additional 5-Fr sheath was inserted in the ipsilateral superficial femoral artery to avoid limb ischemia. An intra-aortic balloon pump was used in seven patients (21\%) to unload the left ventricle. All patients were anticoagulated with intravenous heparin to achieve an activated partial thromboplastin time of $150 \%$ to $200 \%$ that of control. In cases of life-threatening bleeding, heparin could be stopped for a few hours.

Statistical analysis: Pre- and post-transplantation characteristics were compared between the two groups (i.e. dBTT and non-BTT) using Student's t-test for continuous variables (expressed as a mean \pm standard deviation (SD) and Fisher's exact test for categorical variables (expressed as a percentage). A bilateral $P$-value $<0.05$ was considered statistically significant. Survival analysis was performed using the KaplanMeier method. Overall survival and conditional survival after hospital discharge were compared between the two groups using the log-rank test. Overall survival also was analyzed, according to the number of AOF and to the MELD-XI score before HT. To determine predictors of in-hospital mortality, all preoperative variables were analyzed in univariate analysis and were considered for inclusion in a multivariate model (e.g. recipient age, total bilirubin, glomerular filtration rate, MELD-XI score, graft ischemia time, donor age, previous sternotomy, and VA-ECMO at the time of HT). A threshold of $P<0.15$ was used to select variables to be included in the multivariate logistic regression. A semi-saturated propensity score was calculated using all variables in Table 1 . An adjusted analysis was performed using a multivariate Cox regression for survival where the propensity score was included as an independent variable.

Statistical analyses were performed using GraphPad Prism (version 6.0; GraphPad Software Inc., La Jolla, CA) and Stata (StataCorp. 2015. Stata Statistical Software: Release 14. College Station, TX: StataCorp LP).

\section{RESULTS}

Patient characteristics before transplantation: Recipient characteristics before HT were compared. (Table 1) Among the 133 patients included in the study, 99 (74\%) were male, and the mean age was $47 \pm 13$ years. All patients had end-stage heart failure secondary to ischemic $(N=47,35 \%)$, dilated $(N=44,33 \%)$, valvular $(N=13,9.7 \%)$, hypertrophic $(N=10,7.5 \%)$, or congenital $(N=8,6 \%)$ cardiomyopathy. Ischemic cardiomyopathy was the main cause of heart failure in the dBTT group and the second cause after dilated cardiomyopathy in the non-BTT group. Patients in the dBTT group showed a more critical condition in the days prior to HT as reflected by the higher rates of acute kidney failure, liver failure, and mechanical ventilation.

In the dBTT group, 20 patients (52\%) were extubated at the time of HT compared with 82 patients $(82 \%)$ in the non-BTT group $(P=0.002)$. Among dBTT patients, median delay between VA-ECMO implantation and listing was three days (range: 0-20 days), while median delay between VA-ECMO implantation and transplantation was eight days (range: $0-35$ days).
Donor characteristics: Donor characteristics are listed in Supplementary Table 1. (Supplementary Table 1) The graft ischemia times, cardiopulmonary bypass times, and distance between the donors' hospitals and the transplantation centers were longer in the dBTT group, because they had a higher priority status. Other donor characteristics were similar between the two groups.

Survival after HT: In-hospital mortality rates were $23 \%$ and $19 \%$ in the dBTT and non-BTT groups, respectively $(P=$ $0.58)$. As shown in Table 2, the main cause of death was multiple organ failure (50\% of deaths), followed by primary graft failure (29\%), septic shock (21\%), and major bleeding (15\%); there were no differences in the cause of death between the two groups. (Table 2) One-year and five-year overall survival rates were $73.5 \%$ and $62.0 \%$, respectively, in the $\mathrm{dBTT}$ group compared with $80.6 \%$ and $75.5 \%$ in the non-BTT group $(P=0.74)$ (Supplementary Figure 2A). (Supplementary Figure 2) After 10 years, the overall survival was estimated to be $62 \%$ in both groups. Of patients discharged alive from the hospital, the estimated one-year and five-year survival rates were $96.1 \%$ and $81.2 \%$, respectively, in the dBTT group compared with $98.7 \%$ and $92.2 \%$ in the non-BTT group $(P=0.89)$ (Supplementary Figure $2 \mathrm{~B}$ ). In the adjusted analysis, there were no differences in survival between the two groups (hazard ratio $(\mathrm{HR})=0.76$, [95\% confidence interval $(\mathrm{CI})=0.30-1.93], P=0.57)$.

To assess the impact of AOF on post-transplantation survival, patients in both study groups were subdivided according to the presence or absence of AOF at the time of HT. (Figure 1) The lowest survival was observed in dBTT patients with AOF, whereas survival of dBTT patients without AOF was better. In dBTT patients, all but one patient without AOF (dBTT no AOF) survived the study period (one-year survival: $100 \%$; five-year: $100 \%$ ) compared with patients with AOF $(\mathrm{dBTT} \mathrm{AOF}+$ ) who had poor survival rates (one-year: $64 \%$; five-year: $49 \%)(P=0.03)$. The impact of AOF on survival was less important in the non-BTT group: five-year survival rates were $70 \%$ and $80 \%$ in patients with and without AOF, respectively $(P=0.16)$. Patients also were subdivided in three groups, according to their preoperative MELD-XI score: low score $(\leq 10.5)$, intermediate score (10.6-16.4), and high score $(\geq 16.5)$. In-hospital mortality rates were higher and ten-year survival rates lower in patients with MELD-XI score greater than 16.5 in both the dBTT and non-BTT groups (Figure 2A and 2B). (Figure 2)

In univariate analysis, total bilirubin, serum creatinine, MELD-XI score, graft ischemia time, and previous cardiac surgery were independently associated with increasing inhospital mortality. In multivariate analysis, MELD-XI score and previous cardiac surgery remained significant predictors. (Supplementary Table 2)

Hospital outcomes and complications: Lengths of hospitalization, intensive care unit (ICU) stay, and mechanical ventilation times were longer in the $\mathrm{dBTT}$ group (Table 2). When compared with the non-BTT group, patients in the dBTT group had higher rates of primary graft dysfunction $(55 \%$ vs. $32 \%, P=0.01)$ and right ventricular failure $(41 \%$ vs. $27 \%, P=0.04)$. VA-ECMO was required after HT in $50 \%$ of dBTT patients compared with $20 \%$ of non-BTT patients $(P$ 
Table 2. Post-transplantation outcomes during hospitalization

\begin{tabular}{|c|c|c|c|}
\hline Length of stay in ICU, days & $8 \pm 7$ & $7 \pm 6$ & 0.46 \\
\hline Duration of mechanical ventilation, days & $7.8 \pm 1.8$ & $6.4 \pm 3.8$ & 0.79 \\
\hline Duration of any inotropic support, days & $7 \pm 3$ & $7 \pm 4$ & 0.89 \\
\hline In-hospital mortality & $8(23)$ & $19(19)$ & 0.58 \\
\hline Death under VA-ECMO & $1(3)$ & $14(14)$ & 0.001 \\
\hline \multicolumn{4}{|l|}{ Causes of death } \\
\hline Multiple organ failure & $7(20)$ & $12(12)$ & 0.24 \\
\hline Primary graft failure & $1(3)$ & $10(10)$ & 0.48 \\
\hline Stroke & $1(3)$ & 0 & 0.09 \\
\hline Unknown & $1(3)$ & $2(2)$ & 0.75 \\
\hline \multicolumn{4}{|l|}{ Other complications } \\
\hline Acute kidney injury, n (\%) & $24(70)$ & $47(47)$ & 0.03 \\
\hline Temporary hemodialysis, $\mathrm{n}(\%)$ & $9(26)$ & $27(27)$ & 0.92 \\
\hline Maximum level of creatinine, $\mathrm{mmol} / \mathrm{L}$ & $239 \pm 122$ & $212 \pm 134$ & 0.31 \\
\hline Minimum GFR, $\mathrm{ml} / \mathrm{min} / \mathrm{m}^{2}$ & $37 \pm 3$ & $48 \pm 3$ & 0.08 \\
\hline Pneumonia & $11(32)$ & $16(16)$ & 0.04 \\
\hline Stroke & $4(11)$ & $4(4)$ & 0.11 \\
\hline VA-ECMO after heart transplantation & $17(50)$ & $20(20)$ & 0.001 \\
\hline Central & $1(3)$ & $10(10)$ & \\
\hline Peripheral & $16(47)$ & $10(10)$ & \\
\hline Acute respiratory syndrome & $3(8)$ & $13(13)$ & 0.65 \\
\hline
\end{tabular}

Data are mean $\pm \mathrm{SD}$ or $\mathrm{n}(\%)$. Bold font indicates statistical significance.

$=0.001)$. In these cases, femoral cannulas were left in place in 16 out of 17 patients to avoid central cannulation and chest reopening. All patients who required post-HT VA-ECMO in the dBTT group were supported immediately after weaning of cardiopulmonary bypass, whereas eight patients in the non-BTT group were supported within the next few hours. The mean duration of mechanical support was $5.3 \pm 3.9$ days. The mortality under mechanical support was higher in the non-BTT group ( $14 \%$ vs. $1 \%, P=0.001)$.

As listed in Table 2, patients transplanted in the dBTT group had higher rates of reoperation for bleeding $(P=0.01)$, acute kidney injury $(P=0.03)$, and pneumonia $(P=0.04)$ after HT. (Table 2) There were no differences in graft rejection between the two groups. Because patients in the dBTT group had a higher risk of postoperative infection, the use of immunosuppressive induction therapy was lower when compared with the non-BTT group $(72 \%$ versus $88 \%(P=0.04)$ at the Rennes Hospital - data from MHI were not available). The use of rabbit anti-thymocyte globulin (rATG) was less frequent in the dBTT group ( $45 \%$ vs $70 \%, P=0.02)$. 
Supplementary Table 2. Univariate analysis and multivariate Cox regression analysis

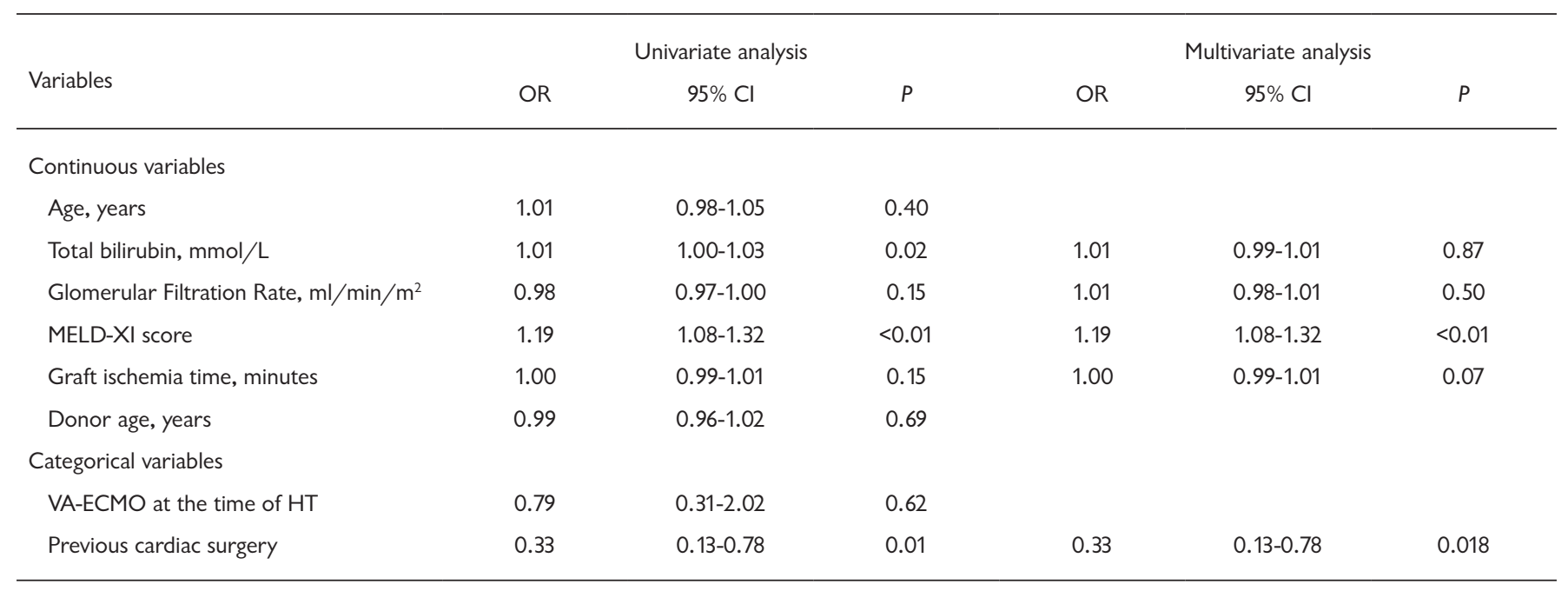

$\mathrm{Cl}$, confidence interval; OR, odds ratio

\section{DISCUSSION}

In this study, the characteristics and outcomes of patients on VA-ECMO support directly bridged to HT (dBTT group) were reviewed in two adult cardiac surgery centers. We compared these results with those of patients who underwent urgent HT without VA-ECMO support at the time of HT (non-BTT group). We found that dBTT patients more likely had ischemic cardiomyopathy, acute kidney failure, respiratory failure, higher MELD-XI score before HT, and longer graft ischemia times. Post-transplant complication rates were higher in $\mathrm{dBTT}$ patients, especially for mechanical circulatory support and reoperation for bleeding. In-hospital mortality was not significantly higher in the dBTT group, and the difference in ten-year conditional survival after hospital discharge also was not significant regardless of adjustment. This may well be due to a lack of statistical power in this study and within the limits of the small sample size, there does not seem to be a gross difference in survival. Moreover, patients with one or more AOF at the time of HT had a lower survival rate in the dBTT group. Finally, MELD-XI score and previous cardiac surgery were associated with an increased in-hospital mortality in both study groups.

The upcoming increase in HT candidates bridged with VA-ECMO: According to European, Canadian, and American guidelines for management of heart failure [Haddad 2009; Ezekowitz 2017; Ponikowski 2016], the use of temporary MCS systems (e.g. VA-ECMO, intra-aortic balloon pump, percutaneous LVAD) should be considered early in the setting of severe, refractory cardiogenic shock (e.g. INTERMACS levels 1 and 2). The choice of the device and the timing of its implantation are based on patient characteristics, operator ability, physician clinical judgment, and optimal degree of hemodynamic support [Mandawat 2017]. Like in many centers, VA-ECMO is the first-line device to support patients with refractory cardiogenic shock in our departments. According to Sauer et al., the rate of VA-ECMO cases in the United States increased by $433 \%$ from 11.4 in 2006 to 60.9 cases per million adult patient discharges in 2011 [Sauer 2015]. With the increase of VA-ECMO use and recent changes in graft allocation policy in the United States, the number of dBTT candidates with VA-ECMO inevitably will increase [McCarthy 2015; Ouyang 2018; Sauer 2015; Paden 2013]. The incidence of VA-ECMO use as a BTT varied from $0.4 \%$ in the United Network for Organ Sharing [Fukuhara 2018; Zalawadiya 2017] to 9.2\% in France [Jasseron 2016], showing the large discrepancy between countries and graft allocation systems. In this series, $11 \%(34 / 296)$ of transplanted patients had VA-ECMO support at the time of HT. In France and Canada, patients supported by VA-ECMO had been assigned the highest priority for nearly 20 years [Jasseron 2016; Haddad 2009]. Recently, the United Network for Organ Sharing implemented a new adult heart graft allocation policy. In this novel 6-status system, the highest priority (status 1) is given to candidates supported with VA-ECMO or surgical biventricular support devices.

The dilemma of dBTT strategy: In the context of a worldwide organ shortage, graft allocation must most importantly maximize the benefits to patients. It is not clear if society should privilege patients with a high risk of mortality on the waiting list or patients with a low risk of post-transplant mortality [Attisani 2012]. In other words, the balance between the risk of death on the waiting list and risk of death after transplantation is not the same across allocation systems. While the need for circulatory support before HT is associated with an increase in post-transplant mortality [Jasseron 2016, Singh 2014], HT provides a survival benefit to patients supported with VA-ECMO. From the Organ Procurement and Transplantation Network database, Singh et al. demonstrated that patients with the highest risk of mortality on the waiting list, such as patients on VA-ECMO, were the ones who drew a greater survival benefit from HT [Singh 2014]. In a study of the French "Cristal" database, Jasseron et al. 

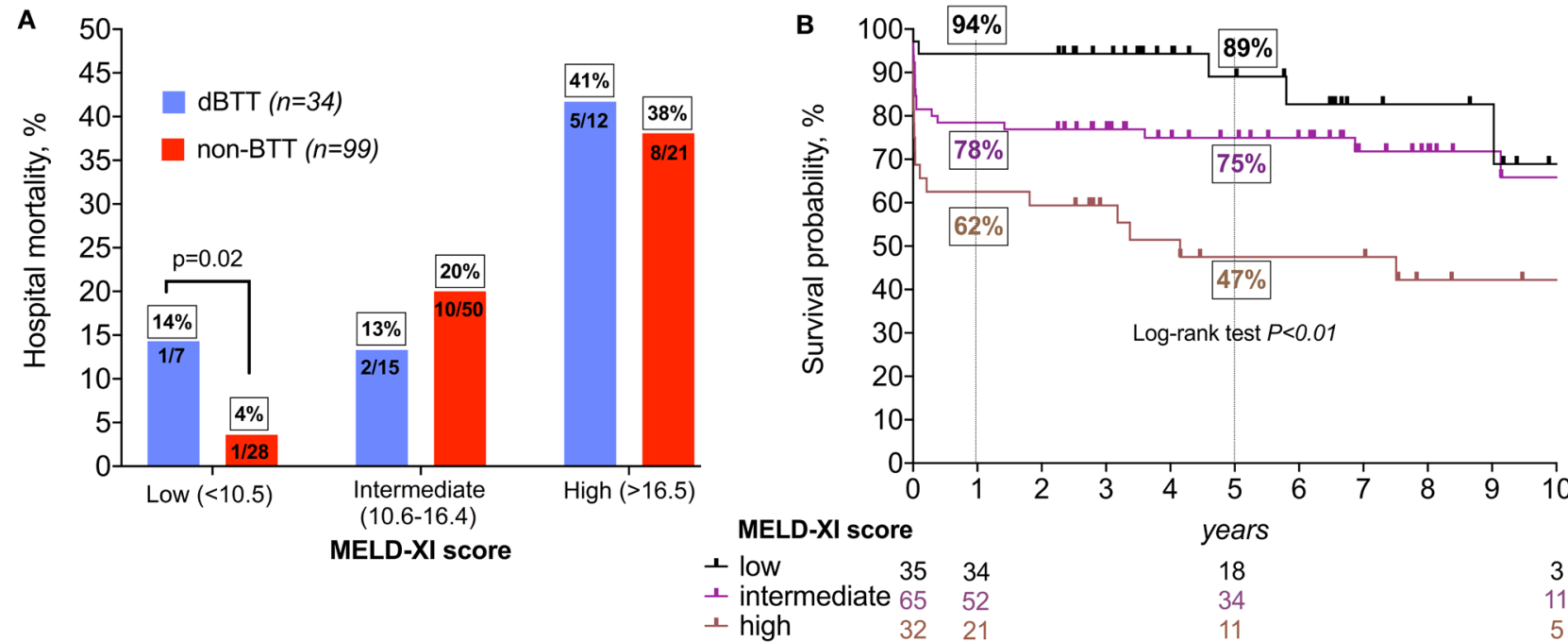

Figure 2. (A) In-hospital mortality in the dBBT and non-BTT group, according to the MELD-XI score. Patients with a high MELD-XI score had a very high mortality rate in both groups. (B) Long-term survival, according to the MELD-XI score. High MELD-XI score was associated with decreased survival in the entire cohort.
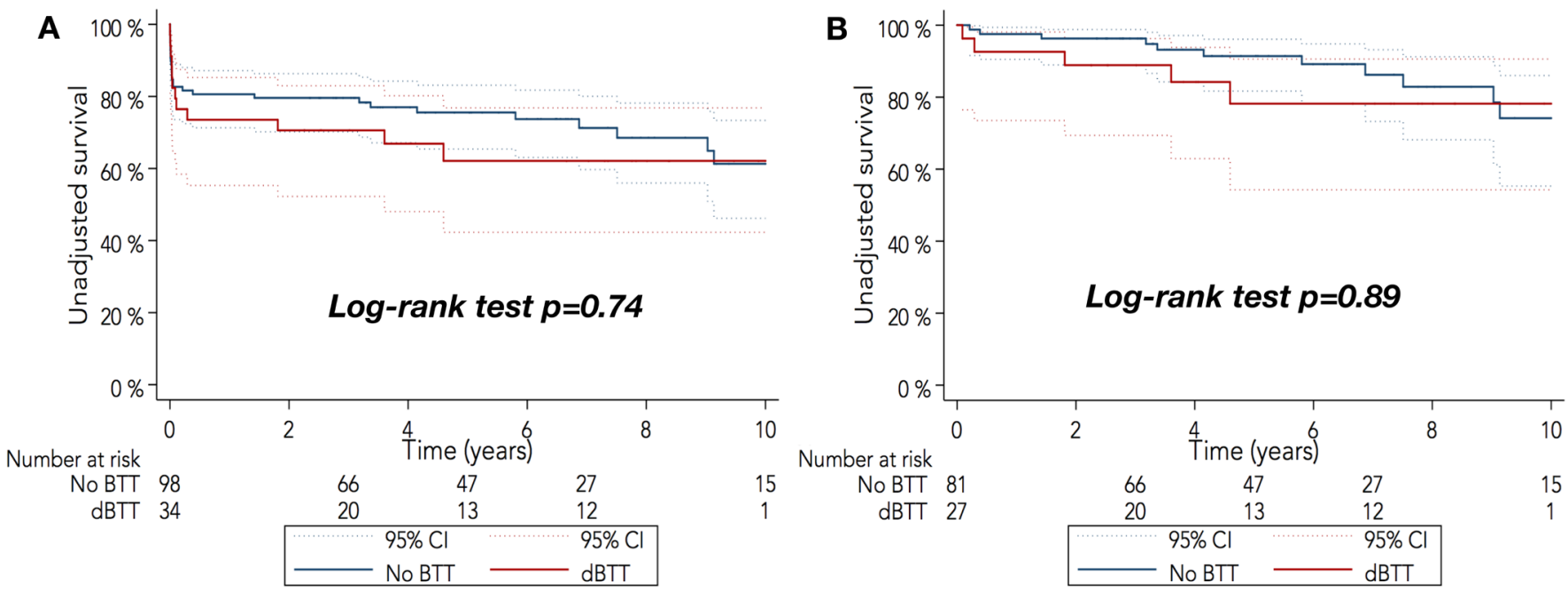

Supplementary Figure 2. Kaplan-Meier curves with $95 \%$ confidence interval for survival after urgent heart transplantation in the two institutions. (A) Overall survival after urgent heart transplantation in patients supported by VA-ECMO (dBTT group) and in patients without VA-ECMO support (non-BTT group).

(B) Conditional survival after hospital discharge between the two groups.

showed that HT provides a survival benefit for patients supported by VA-ECMO at the time of transplant listing [Jasseron 2016]. They also reported a one-year survival of $70.4 \%$, which is higher than other large series.

In our experience, one-year survival was $74 \%$ for all patients and was higher in patients without any AOF. The MELD-XI score also appears to identify patients at high risk for mortality after HT [Fukuhara 2018]. The suggestion that AOF should be examined as a predictor of post-transplant outcomes had previously been presented by Cho et al. [Cho 2015]. They demonstrated that the use of organ failure scores, particularly the MELD UNOS score, had a better predictive value than duration of ECMO. There was a large requirement for VAECMO post-HT in these patients. This is mostly explained by a more liberal use of prolonged support in the presence of multiple risk factors for primary graft dysfunction. In these patients, VA-ECMO was pre-emptively kept post-HT.

Despite acceptable results, post-transplant survival was lower in $\mathrm{dBTT}$ patients than in non-bridged patients, mostly because of excess early mortality, which is consistent with most previously published studies [Jasseron 2016; Barth 2012; Cho 2015; Fukuhara 2018; Zalawadiya 2017; BargeCaballero 2014; Chung 2009]. Indeed, one-year survival in patients on VA-ECMO support directly bridged to HT varies 
from 57.8\% to 72\% [Jasseron 2016; Cho 2015; Fukuhara 2018; Zalawadiya 2017]. Recently, Ouyang et al. reviewed the incidence and outcomes of HT in patients with temporary MCS using the National Inpatient Sample [Ouyang 2018]. They showed that the use of temporary MCS before HT is increasing $(6.6 \%$ of $\mathrm{HT})$ with a trend toward decreasing posttransplant mortality over time.

LVAD implantations in "crash and burn" patients mostly have been abandoned, due to poor survival [Shah 2017; Kirklin 2014]. The use of percutaneous LVAD devices, such as the Impella device, opens the door to other types of dBTT with good results [Lima 2016; Seese 2020] and occasionally lower complication rates compared with VA-ECMO [Lemor 2020]. Patients on VA-ECMO, however, are still likely to have had a worse hemodynamic compromise, and VA-ECMO is prioritized in institutions for patients with INTERMACS 1 or patients who likely will have persisting poor oxygenation [Garan 2019]. However, it is not clear if hemodynamic stabilization with temporary MCS in these critically ill patients improves outcomes after LVAD implantation, and the bridgeto-bridge strategy still is up for debate in the literature [Attisani 2012]. Our results suggest that we might consider HT in candidates on VA-ECMO if full recovery of end-organ functions is observed within one or two weeks following circulatory support initiation.

Limitations: The present study is retrospective and includes a small number of patients from the two participating institutions. Due to this small sample size, it is not possible to comment on the difference in in-hospital mortality and conditional mortality between the dBTT and non-BTT groups. The differences remained not statistically significant, but whether a possible clinical significance can be drawn is beyond the capacity of the data presented here, though there does not seem to be gross differences. There also are institutional differences in clinical decision-making with regard to the management of patients in cardiogenic shock and to the subsequent decision to offer HT to patients on VA-ECMO. For this reason, the impact of differing medical management on outcomes cannot be ruled out.

\section{CONCLUSIONS}

Patients on VA-ECMO, specifically those without any AOF, remain good candidates for HT with low in-hospital mortality and adequate long-term survival. A MELD-XI score greater than 16.5 was associated with higher in-hospital mortality following HT and should constitute a contraindication.

\section{REFERENCES}

Agence de la Biomédecine. 2016. Rapport Médical et Scientifique de l'Agence de La Biomédecine - Greffe Cardiaque.

Attisani M, Centofanti P, Torre ML, et al. 2012. Advanced heart failure in critical patients (INTERMACS 1 and 2 levels): ventricular assist devices or emergency transplantation? Interact Cardiovasc Thorac Surg. 15(4):678-684.
Barge-Caballero E, Almenar-Bonet L, Villa-Arranz A, et al. 2014. Impact of short-term mechanical circulatory support with extracorporeal devices on postoperative outcomes after emergency heart transplantation: Data from a multi-institutional Spanish cohort. Int J Cardiol. 176(1):86-93.

Barth E, Durand M, Heylbroeck C, et al. 2012. Extracorporeal life support as a bridge to high-urgency heart transplantation: ECLS and heart transplantation. Clin Transplant. 26(3):484-488.

Cho Y hyun, Yang J, Sung K, et al. 2015. Extracorporeal Life Support as a Bridge to Heart Transplantation: Importance of Organ Failure in Recipient Selection. ASAIO J. 61(2):139-143.

Chung JC-Y, Tsai P-R, Chou N-K, Chi N-H, Wang S-S, Ko W-J. 2009. Extracorporeal membrane oxygenation bridge to adult heart transplantation: ECMO bridge to heart transplantation. Clin Transplant. 24(3):375-380.

Dardas TF. 2018. Temporary circulatory support devices as a bridge to transplant: Boon or bane? J Heart Lung Transplant. 37(9):1045-1046.

Ezekowitz JA, O'Meara E, McDonald MA, et al. 2017. 2017 Comprehensive Update of the Canadian Cardiovascular Society Guidelines for the Management of Heart Failure. Can J Cardiol. 33(11):1342-1433.

Fukuhara S, Takeda K, Kurlansky PA, Naka Y, Takayama H. 2018. Extracorporeal membrane oxygenation as a direct bridge to heart transplantation in adults. J Thorac Cardiovasc Surg. 155(4):1607-1618.e6.

Garan AR, Takeda K, Salna M, et al. 2019. Prospective Comparison of a Percutaneous Ventricular Assist Device and Venoarterial Extracorporeal Membrane Oxygenation for Patients With Cardiogenic Shock Following Acute Myocardial Infarction. J Am Heart Assoc Cardiovasc Cerebrovasc Dis. $8(9)$.

Grimm JC, Shah AS, Magruder JT, et al. 2015. MELD-XI Score Predicts Early Mortality in Patients After Heart Transplantation. Ann Thorac Surg. 100(5):1737-1743.

Haddad H, Isaac D, Legare J, et al. 2009. Canadian Cardiovascular Society Consensus Conference update on cardiac transplantation 2008: Executive Summary. Can J Cardiol. 25(4):197-205.

Isaac D, Chan M, Haddad H, et al. 2011. Cardiac Transplantation: Eligibility and Listing Criteria in Canada 2012.

Jasseron C, Lebreton G, Cantrelle C, et al. 2016. Impact of Heart Transplantation on Survival in Patients on Venoarterial Extracorporeal Membrane Oxygenation at Listing in France: Transplantation. 100(9):1979-1987.

Kirklin JK, Naftel DC, Pagani FD, et al. 2014. Sixth INTERMACS annual report: A 10,000-patient database. J Heart Lung Transplant. 33(6):555-564.

Lemor A, Hosseini Dehkordi SH, Basir MB, et al. 2020. Impella Versus Extracorporeal Membrane Oxygenation for Acute Myocardial Infarction Cardiogenic Shock. Cardiovasc Revasc Med. 21(12):1465-1471.

Levy B, Bastien O, Karim B, et al. 2015. Experts' recommendations for the management of adult patients with cardiogenic shock. Ann Intensive Care. 5(1):52.

Lima B, Kale P, Gonzalez-Stawinski GV, Kuiper JJ, Carey S, Hall SA. 2016. Effectiveness and Safety of the Impella 5.0 as a Bridge to Cardiac Transplantation or Durable Left Ventricular Assist Device. Am J Cardiol. 117(10):1622-1628.

Mandawat A, Rao SV. 2017. Percutaneous mechanical circulatory support devices in cardiogenic shock. Circ Cardiovasc Interv. 10(5).

McCarthy FH, McDermott KM, Kini V, et al. 2015. Trends in U.S. 
Extracorporeal Membrane Oxygenation Use and Outcomes: 2002-2012. Semin Thorac Cardiovasc Surg. 27(2):81-88.

Mehta RL, Kellum JA, Shah SV, et al. 2007. Acute Kidney Injury Network: report of an initiative to improve outcomes in acute kidney injury. Crit Care. 11(2):R31.

Muller G, Flecher E, Lebreton G, et al. 2016. The ENCOURAGE mortality risk score and analysis of long-term outcomes after VA-ECMO for acute myocardial infarction with cardiogenic shock. Intensive Care Med. 42(3):370-378.

Ouyang D, Gulati G, Ha R, Banerjee D. 2018. Incidence of temporary mechanical circulatory support before heart transplantation and impact on post-transplant outcomes. J Heart Lung Transplant. 37(9):1060-1066.

Paden ML, Conrad SA, Rycus PT, Thiagarajan RR. 2013. Extracorporeal Life Support Organization Registry Report 2012. ASAIO J. 59(3):202-210.

Ponikowski P, Voors AA, Anker SD, et al. 2016. 2016 ESC Guidelines for the diagnosis and treatment of acute and chronic heart failureThe Task Force for the diagnosis and treatment of acute and chronic heart failure of the European Society of Cardiology (ESC)Developed with the special contribution of the Heart Failure Association (HFA) of the ESC. Eur
Heart J. 37(27):2129-2200.

Sauer CM, Yuh DD, Bonde P. 2015. Extracorporeal Membrane Oxygenation Use Has Increased by $433 \%$ in Adults in the United States from 2006 to 2011: ASAIO J. 61(1):31-36.

Seese L, Hickey G, Keebler ME, et al. 2020. Direct bridging to cardiac transplantation with the surgically implanted Impella 5.0 device. Clin Transplant. 34(3):e13818.

Shah P, Pagani FD, Desai SS, et al. 2017. Outcomes of Patients Receiving Temporary Circulatory Support Before Durable Ventricular Assist Device. Ann Thorac Surg. 103(1):106-112.

Singh TP, Milliren CE, Almond CS, Graham D. 2014. Survival Benefit From Transplantation in Patients Listed for Heart Transplantation in the United States. J Am Coll Cardiol. 63(12):1169-1178.

The International Society for Heart and Lung Transplantation. 2018. International Thoracic Organ Transplant (TTX) Registry Data Slides Overall Heart Transplantation Statistics.

Zalawadiya S, Fudim M, Bhat G, Cotts W, Lindenfeld J. 2017. Extracorporeal membrane oxygenation support and post-heart transplant outcomes among United States adults. J Heart Lung Transplant. 36(1):77-81. 